\title{
Effects of the combined PNF and deep breathing exercises on the ROM and the VAS score of a frozen shoulder patient: Single case study
}

\author{
Byung-Ki Lee* \\ Department of Physical Therapy, Daewon University College, Jecheon, Korea
}

This study was conducted to examine the influence of combined exercise using proprioceptive neuromuscular facilitation (PNF) and deep breathing exercise on range of motion (ROM) and visual analog scale (VAS) score in acute frozen shoulder patient. The subject of this study was woman complained disabilities in daily routine due as a frozen left shoulder. The exercise program was composed of 11 sessions and continued four weeks. The program was composed of PNF and deep breathing exercise, and the subject was compared by passive ROM (shoulder flexion, abduction, and internal and external rotation) test and
VAS score in shoulder movement before and after the exercise. The results showed that patient who practiced this program, the ROM of the shoulder joint increased and the VAS score decreased. Thus, this program was shown to be effective in suppressing pain and increasing the ROM of the shoulder joint in acute frozen shoulder patient.

Keywords: Frozen shoulder, Proprioceptive neuromuscular facilitation, Deep breathing exercise, Closed kinetic chain exercise, Range of motion, Visual analog scale

\section{INTRODUCTION}

Adhesive shoulder capsulitis, widely known as a degenerative disease, is commonly observed among peoples in 40-60 yr old, and it is known as "frozen shoulder" (Shanahan and Sladek, 2011). The incidence of frozen shoulder is increasing in younger generations. Frozen shoulder limits the daily activities of the patients by causing pain around their shoulder and reducing the range of motion (ROM) of their joints. Limitation of ROM is due to the adhesion of the joints, hyperplasia of the synovial membranes through reduction of the area of the joint cavity, contraction of the articular capsule, and historical tissue proliferation (Akpinar, et al., 2003; Loew et al., 2005). Frozen shoulder especially decreases the abduction, internal rotation, and external rotation of the shoulder and the patients in the initial phase complain of limitation in their activities and pain in their shoulder joints (Hamdan et al., 2003).

The progression of a frozen shoulder is divided into the freezing phase, the frozen phase, and the thawing phase. Partial or complete functions are usually recovered in one to three years, because the disorder is accompanied by severe limitations in activities (Costic et al., 2003). Many people with frozen shoulders are also known to suffer not only limitations in activities but also night pain. Such pain is often chronic, which degrades the patient's quality of life and ability to perform his or her daily routine (Cho et al., 2002). Such musculoskeletal system pain results in motor control disorders and degrades the balance control capability. These can cause secondary problems and weakening (Kim et al., 2011), as well as psychological problems that will further limit the movements and weaken the muscles (You and Jung, 2001).

The proprioceptive neuromuscular facilitation (PNF), developed by Knott and Kabat, is a type of therapeutic exercise composed of a pattern of muscular contractions and diagonal movements that are effective on paralyzed muscles and pain of muscles. (Bae et al., 1993).

The effectiveness of the rehabilitation program for a frozen shoul-
${ }^{*}$ Corresponding author: Byung-Ki Lee

Department of Physical Therapy, Daewon University College, 316 Daehak-ro, Jecheon 27135, Korea

Tel: +82-43-649-3159, Fax: +82-43-649-3690, E-mail: toppt@naver.com

Received: August 25, 2015 / Accepted: October 1, 2015
This is an Open Access article distributed under the terms of the Creative Commons Attribution Non-Commercial License (http://creativecommons.org/licenses/by-nc/3.0/) which permits unrestricted non-commercial use, distribution, and reproduction in any medium, provided the original work is properly cited. 
der patient consisted of reduction pain, improving ROM, and other therapeutic benefits. Moreover, the deep breathing exercise and the relaxation exercises are now recognized as effective treatment methods that improve the daily life of the patient by reducing his or her pain (Friesner et al., 2006; Schaffer and Yucha, 2004).

This study was conducted to examine the influence of a combined exercise regimen of indirect treatment using PNF and deep breathing exercise of the closed kinetic chain on the visual analog scale (VAS) and ROM in frozen shoulder patient.

\section{MATERIALS AND METHODS}

\section{Participants}

The subject was a 46 yr-old woman who visited a hospital in J city in Korea due to prolonged labor that involved movements related to lifting heavy objects. She was diagnosed with adhesive capsulitis (Table 1). She was a housewife who complained difficulty in performing housework, especially cleaning, dishwashing, and placing or retrieving objects in or from a high position. Due to the adhesive capsulitis in the left shoulder, the movement range of the left shoulder joint was limited. The flexion limitation was 100 degree and the abduction limitation was 60 degree. The limitation in the external rotation was 40 degree and the limitation in the internal rotation was 25 degree. The level of pain when the arm was moved was around 7 in the VAS.

\section{Instrument}

A goinometer was used to measure the ROM, and VAS was used to check the level of improvement of the pain felt when the shoulder joint was moved. The tests were conducted before the starting of exercise and three weeks after the last session, three times per session.

\section{Measurement of the range of motion of the shoulder joint}

The ROM in the shoulder joint was measured using a goniometer, according to the instructions of the American Medical Association, to measure the flexion, abduction, and internal and external rotations, which were subject to limitations (American Medical Association, 1993). With the patient in the supine position,

Table 1. Characteristics of the participant

\begin{tabular}{lccccc} 
Sex & Age & Height & Weight & Diagnosis & $\begin{array}{c}\text { Rehabilitation } \\
\text { period }\end{array}$ \\
\hline Female & 46 & 153 & 52 & $\begin{array}{c}\text { Adhesive capsuli- } \\
\text { tis of left shoulder }\end{array}$ & 4 weeks \\
\hline
\end{tabular}

the angle at the point of limitation of the joint movement was measured, using the range of the manual movement. Two measurements were taken for each movement, and the average of the two values was used. The flexion of the shoulder was measured as the angle of the arm lifted vertically, and the abduction movement was measured at the angle of the arm spread sideways from the position in which the upper trunk was fixed and the palm side of the arm was touching the body. The internal and external rotations were measured as the maximum angle of movement of the arm toward the head, with the upper trunk fixed, the arm abducted by 90 degree, and the elbow bent by 90 degree.

\section{VAS}

The pain level was measured using VAS, which is widely used in clinical studies to objectively quantify pain severity. Ibrahim et al. (2014) also used VAS in their study to evaluate the level of pain felt by people with adhesive capsulitis in the shoulder, after stretching sessions.

The VAS used in this study was composed of a $100 \mathrm{~mm}$-long straight line, in which the left end $(0 \mathrm{~mm})$ indicated a state free of pain, and the right end $(100 \mathrm{~mm})$, the maximum pain. Then the level of pain was checked during the manual movement of the joints for the ROM measurement, along a straight line from 0 $\mathrm{mm}$ to $100 \mathrm{~mm}$, where the scale increased by 1 every $10 \mathrm{~mm}$.

\section{Procedure}

The procedure of this study was composed of four phases. In the first phase, the patient was evaluated and plans were made. In the second phase, preliminary tests were conducted. In the third phase, the combined exercise scheme was applied. In the fourth final phase, the following tests were performed. The combined exercise program in the third phase was performed over a threeweek period, in 11 sessions from April 2, 2014 to April 25, 2014. Each of these sessions lasted $30 \mathrm{~min}$. While the program was in progress, the patient was given two- to three-minute breaks depending on pain level. Also, after each program, a five-minute break was offered. The design of this rehabilitation exercises process is shown in Table 2 .

\section{Patient evaluation and treatment planning}

PNF includes the international classifications of functionality, disability, and health (ICF). It was introduced by WHO in the planning phase of the treatment to improve activity limitation in daily life. The subject was interviewed before the exercise and the subjective elements that she felt were noted. The patient could 
Table 2. PNF programs

\begin{tabular}{clll}
\hline Session & Major training content & Significances and tests conducted & Supporting material \\
\hline 1 & Pre test & PROM, Pain test with VAS & \\
2 & Orientation & Deep breathing & Electric table \\
3 & Exercise for flexion & PWB-CKCE with DB & Electric table \\
4 & Exercise for flexion & PWB-CKCE with DB & Electric table \\
5 & Exercise for flexion & FWB-CKCE & Wall \\
& Exercise for abduction & PWB-CKCE with DB & Electric table \\
6 & Exercise for flexion & FWB-CKCE & Wall \\
7 & Exercise for abduction & PWB-CKCE with DB & Electric table \\
& Exercise for abduction & FWB-CKCE & Electric table \\
8 & Exercise for rotation & PWB-CKCE with DB & Electric table \\
& Exercise for flexion & FWB-CKCE & Electric table \\
& Exercise for abduction & FWB-CKCE & Electric table \\
9 & Exercise for rotation & PWB-CKCE with DB & Electric table \\
& Exercise for flexion & FWB-CKCE & Electric table \\
10 & Exercise for abduction & FWB-CKCE & Electric table
\end{tabular}

PNF, Proprioceptive neuromuscular facilitation; PWB-CKCE, partial weight bearing-closed kinetic chain exercise; FWB-CKCE, full weight bearing-closed kinetic chain exercise; $\mathrm{DB}$, deep breathing; VAS, visual analog scale.

not perform her daily routine, such as cleaning, wearing a bra, or raising/lowering her arm, and she complained difficulty and pain. When the ROM test was conducted, limitation in flexion, abduction, internal rotation, and external rotation was observed.

\section{Baseline tests and follow-up tests}

The baseline test was conducted before the start of the exercise program. The test included the manual ROM and the VAS. The follow-up test was conducted three weeks after all the programs were completed, using the same ROM and VAS methods.

\section{Combined PNF and deep breathing exercise program}

The exercise program was composed of 11 sessions, which included the baseline test and the follow-up test. To improve the $\mathrm{ROM}$ in the shoulder joint, the sessions were conducted in the following order: flexion improvement, abduction, internal rotation, and external rotation of the shoulder.

The exercises combined partial body weight support and deep breathing. They included the full body weight load and the indirect treatment, which involved moving the shoulder through the resistant exercise. For the exercise segments in the program, a total of eight sessions were held over three weeks, at three sessions a week. Each session lasted $30 \mathrm{~min}$. In the second session before the start of the all-out exercises, the patients were briefed on the progress of the overall exercises and on how to perform a deep breath- ing exercise. When the patient complained pain, the exercise was stopped and a two- to three-minute break was offered. These breaks were not included in the duration of the exercises.

\section{Data analysis}

The collected measurement data were presented through a visual analysis. The data before the exercise and four weeks after the exercise were plotted using MS Office Excel 2007 software. All the values used for the plot were averages of the three measurements.

\section{RESULTS}

\section{Results of ROM}

The measurement of the ROM was based on the tests for the manual range of the movements. The measurements were taken in the following order: shoulder flexion, abduction, internal rotation, and external rotation.

The flexion of the shoulder improved from 100 degree in the pre-test before the exercise to 160 degree in the post-test. Likewise, the abduction improved from 60 degree in the pre-test to 110 degree in the post-test. The internal rotation improved from 25 degree to 55 degree, and the external rotation improved from 40 degree to 60 degree (Fig. 1). 


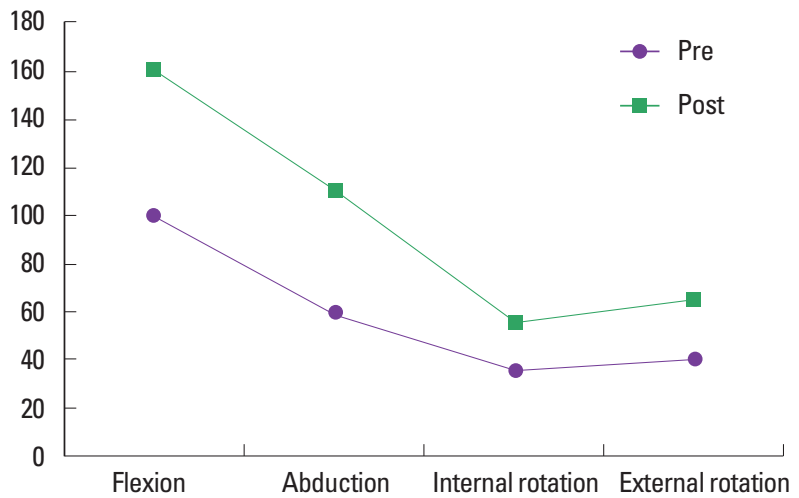

Fig. 1. Result of range of motion (ROM).

\section{Results of VAS}

The level of the subjective pain was measured using VAS when the shoulder joint was moved, with 10 as the highest level of pain. The pain level during flexion of the shoulder before the exercise was 6 , and pain level was reduced to 2 after three weeks of exercise at the maximum angle. In the case of the abduction movement, the pain level improved from 6 before the exercise to 2 after the exercise. The internal and external rotation at the maximum angle, the pain level improved from 7 before exercise to 2 after the exercise (Fig. 2).

\section{DISCUSSION}

Adhesive capsulitis is a musculoskeletal disorder referred to as "frozen shoulder" in clinical practice. It involves adhesion to the surface of the joint due to degenerative changes in the shoulder joint and its surrounding areas. This change is accompanied by significant pain, which leads to gradual reduction of the ROM and the muscular strength around the shoulder, and causes significant impairment in the patient's daily routine (Donatelli et al., 2014). The acute phase patient subject was studied on two months after the occurrence of adhesive capsulitis on her left shoulder. Her VAS score was around 6-7, which induced motion limitation and pain. It is known that a frozen shoulder is caused by inflammation after an irritation around the shoulder, which increases the tension and pain in the muscles that causes them to contract and limited the range of their movement. For this reason, indirect treatment of PNF, closed kinetic chain exercises, and deep breathing exercises have been used to extend the contracted muscles around the shoulder so as to minimize the pain.

Deep and slow breathing exercise is known to be effective in reducing the tension in the muscles of patients who suffer from pain

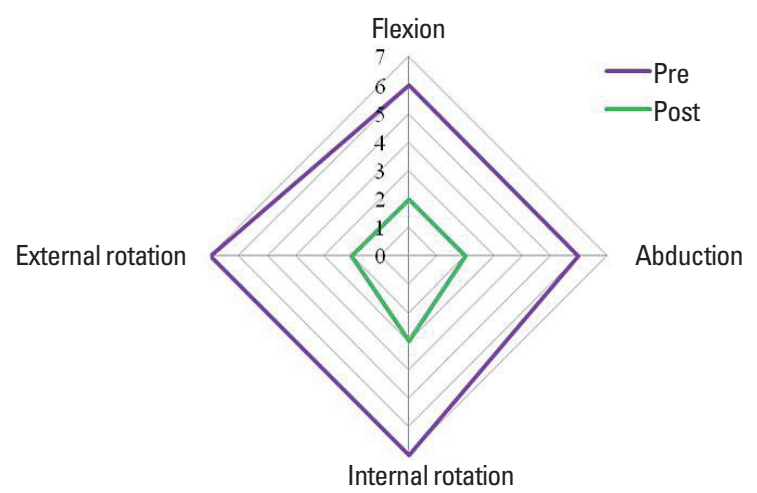

Fig. 2. Result of visual analog scale (VAS).

(Busch et al., 2012). The preceding study of Hyun and Kang (2002) proved that Danjeon breathing exercise is also effective in enhancing the flexibility of and reducing pain in the shoulder of middle-aged female patients. The PNF exercise used in this study was the trunk extension movement during expiration, and the closed kinetic chain exercise was performed as a partial weight-bearing and full-weight-bearing exercise. A closed kinetic chain exercise is a type of movement that takes place when resistance is applied onto the proximal and distal ends at the same time, while the distal ends of the extremities are fixed. The subject of this study, a frozen shoulder patient, suffered from significant pain, as her VAS score was 7. Therefore, a partial weight on distal segments, such as a hand, was applied instead of a full-weight-bearing exercise. Also, as the subject performed a closed kinetic chain exercise, the distal ends were fixed so that the ascent and descent of her trunk during the expiration could accompany the abduction, flexion, and rotation of her shoulder (Fig. 3).

The process was suggested as a mean of strengthening the weakened muscles and performing distal segment movements by controlling the proximal segments such as the trunk using the closed kinetic chain exercise (McMullen and Uhl, 2000). The indirect treatment of the PNF applies the selection of the moved parts on the uninvolved parts of the body to use the principles of the synergetic muscles. That is, using the manual resistance of the therapist, the parts of the body that are not directly in motion are moved indirectly, or contractions of the muscles are generated (Angel and Eppler, 1967; Hellebrandt et al., 1947). The indirect treatment of PNF is based on the irradiation effect, using the positive approach philosophy of PNF and the manual resistance, which is widely used for patients with musculoskeletal disorders. Also, in this study, such a control method for proximal ends was used to combine the movements of the trunk during expiration 

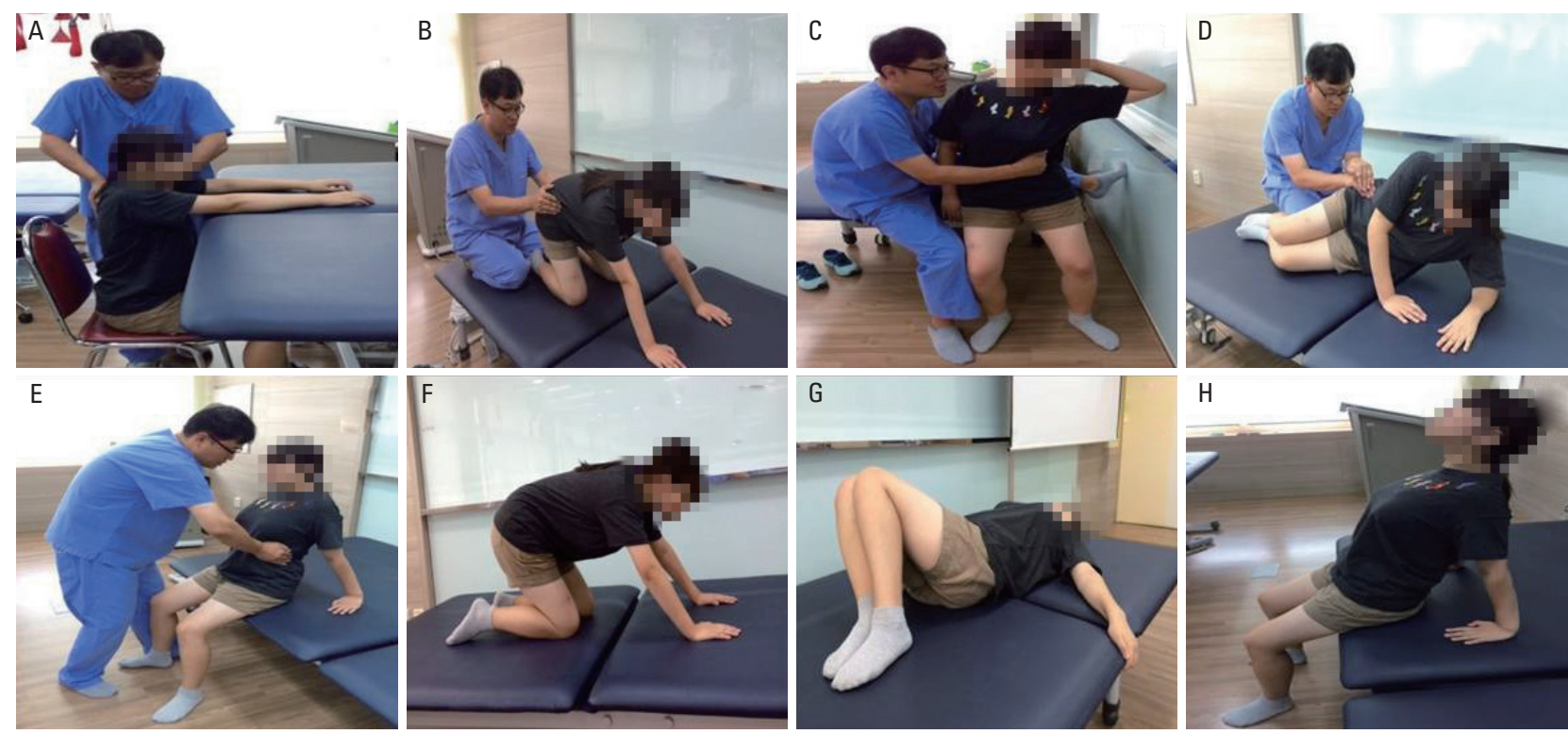

Fig. 3. PNF programs. (A) PWB-CKCE with DB for shoulder flexion; (B) FWB-CKCE for shoulder flexion; (C) PWB-CKCE with DB for shoulder abduction; (D) FWB-CKCE for shoulder abduction; (E) PWB-CKCE with DB for shoulder rotation; (F-H) Home exercises for patient. PNF, Proprioceptive neuromuscular facilitation; PWB-CKCE, partial weight bearing-closed kinetic chain exercise; FWB-CKCE, full weight bearing-closed kinetic chain exercise; DB, deep breathing.

and the closed kinetic chain exercise. For this, the distal end, elbow, was fixed using the body weight of the subject. To reduce the amount of pain due to the body weight, deep breathing and the partial body weight were used at the start of the exercise. Then the posture of the subject was changed so that the load on the distal ends could be increased according to the degree of pain, which would help stabilize the joints during a closed kinetic chain exercise. Iwasaki et al. (2006) showed that the closed kinetic chain exercise was used mainly for muscle strength improvement, wherein the antagonist is applied to the eccentric contraction to yield a significant benefit.

The patient in this study had limited ROM due to the inflammation reaction, such as the contraction of her muscular tissues and pain when she tried to move her joint after the adhesion in her gleno-humeral joint. To minimize the degree of pain felt during the rehabilitation, the indirect method of PNF was used, through the movement of other parts of the body. Also, the deep breathing exercise was accompanied by the closed kinetic chain exercise to decrease the tension of the muscle and to help it relax. This exercise method reduced the pain and increased the ROM effectively.

In this study, the PNF, which was used as a rehabilitation program for frozen shoulder patient, is a mean of facilitating the contraction of the muscles and the movements of the joints. In many preceding studies, people with shoulder disorders were put on stretching programs, with emphasis on the effectiveness of the muscular exercise in the shoulder joints. Cornelius et al. (1995) reported that PNF is an effective treatment that increases the ROM. Al Dajah (2014) recently reported that as the muscle length increased, the scale of the external rotation of the gleno-humeral joint also increased, and PNF was used to reduce the pain.

In this study, a combined regime of indirect treatment using deep breathing exercise with a focus on PNF and closed kinetic chain exercise were used. The subject of this study was an acute phase patient, and exercise rehabilitation program using PNF proved effective in reducing pain and increasing ROM. Therefore, in the follow-up study, more frozen shoulder patients should be included using the same regime.

\section{CONFLICT OF INTEREST}

No potential conflict of interest relevant to this article was reported.

\section{REFERENCES}

Akpinar S, Ozalay M, Hersekli MA, Ozkoc G, Tandogan RN. Arthroscopic capsular release for frozen shoulder. Acta Orthop Traumatol Turc 2003;37:213-218.

Al Dajah SB. Soft tissue mobilization and PNF improve range of motion 
and minimize pain level in shoulder impingement. J Phys Ther Sci 2014;26:1803-1805.

Angel RW, Eppler WG Jr. Synergy of contralateral muscles in normal subject and patients with neurogic disease. Arch Phys Med Rehabili 1967;48:233-239.

Bae SS. A study of proprioceptive neuromuscular facilitation principles. J Korean Phys Soc 1993;5:109-114.

Busch V, Magerl W, Kern U, Haas J, Hajak G, Eichhammer P. The effect of deep and slow breathing on pain perception, autonomic activity, and mood processing--an experimental study. Pain Med 2012;13:215228.

Cho KH, Song JY, Lee H, Kim JS, Rhee YG. The effect of subacromial bursa injection of hyaluronate in patients with adhesive capsulitis of shoulder joint. J Korean Acad Rehabil Med 2002;26:73-80.

Cornelius WL, Jensen RL, Odell ME. Effects of PNF stretching phase on acute arterial blood pressure. Can J Appl Physiol 1995;20:222-229.

Costic RS, Jari R, Rodosky MW, Debski RE. Joint compression alters the kinematics and loading patterns of the intact and capsule-transected AC joint. J Orthop Res 2003;21:379-385.

Donatelli R, Ruivo RM, Thurner M, Ibrahim MI. New concepts in restoring shoulder elevation in a stiff and painful shoulder patient. Phys Ther Sport 2014;15:3-14.

Friesner SA, Curry DM, Moddeman GR. Comparison of two pain-management strategies during chest tube removal: relaxation exercise with opioids and opioids alone. Heart Lung 2006;35:269-276.

Hamdan TA and Al-Essa KA. Manipulation under anaesthesia for the treatment of frozen shoulder. Int Orthop 2003;27:107-109.

Hellebrandt FA, Parrish AM, Hountz SJ. Cross education, the influence of unilateral exercise on the contralateral limb. Arch Phys Med Rehabil
1947;28:76-85.

Hyun KS, Kang HS. Effect of dan-jun breathing exercise program experienced by women in midlife. J Korean Acad Fundam Nurs 2002;9:180189.

Ibrahim M, Donatelli R, Hellman M, Echternach J. Efficacy of a static progressive stretch device as an adjunct to physical therapy in treating adhesive capsulitis of the shoulder: a prospective, randomised study. Physiotherapy 2014;100:228-234.

Iwasaki T, Shiba N, Matsuse H, Nago T, Umezu Y, Tagawa Y, Nagata K, Basford JR. Improvement in knee extension strength through training by means of combined electrical stimulation and voluntary muscle contraction. Tohoku J Exp Med 2006;209:33-40.

Kim SR, Lee JH, Ahn SH. The Effects of a Functional Movement Screen on Pain and Performance Ability in Professional Fencing Players. J Korean Phys Soc 2011;23:21-28.

Loew M, Heichel TO, Lehner B. Intraarticular lesions in primary frozen shoulder after manipulation under general anesthesia. J Shoulder Elbow Surg 2005;14:16-21.

McMullen J, Uhl TL. A kinetic chain approach for shoulder rehabilitation. J Athl Train 2000;35:329-337.

Shanahan EM, Sladek R. Shoulder pain at the workplace. Best Pract Res Clin Rheumatol 2011;25:59-68.

Schaffer SD, Yucha CB. Relaxation \& pain management: the relaxation response can play a role in managing chronic and acute pain. Am J Nurs 2004;104:75-81.

You JE, Jung EH. The effects of traditional physical therapy on pain reduction and depression level of patients with chronic low back pain. J Korean Phys Soc 2001;13:677-683. 